\title{
Statistical patterns in the location of natural lightning
}

\author{
F. G. Zoghzoghy, ${ }^{1}$ M. B. Cohen, ${ }^{1}$ R. K. Said, ${ }^{1,2}$ and U. S. Inan ${ }^{1,3}$ \\ Received 27 September 2012; revised 13 November 2012; accepted 10 December 2012.
}

[1] Lightning discharges are nature's way of neutralizing the electrical buildup in thunderclouds. Thus, if an individual discharge destroys a substantial fraction of the cloud charge, the probability of a subsequent flash is reduced until the cloud charge separation rebuilds. The temporal pattern of lightning activity in a localized region may thus inherently be a proxy measure of the corresponding timescales for charge separation and electric field buildup processes. We present a statistical technique to bring out this effect (as well as the subsequent recovery) using lightning geo-location data, in this case with data from the National Lightning Detection Network (NLDN) and from the GLD360 Network. We use this statistical method to show that a lightning flash can remove an appreciable fraction of the built up charge, affecting the neighboring lightning activity for tens of seconds within a $\sim 10 \mathrm{~km}$ radius. We find that our results correlate with timescales of electric field buildup in storms and suggest that the proposed statistical tool could be used to study the electrification of storms on a global scale. We find that this flash suppression effect is a strong function of flash type, flash polarity, cloud-to-ground flash multiplicity, the geographic location of lightning, and is proportional to NLDN model-derived peak stroke current. We characterize the spatial and temporal extent of the suppression effect as a function of these parameters and discuss various applications of our findings.

Citation: Zoghzoghy, F. G., M. B. Cohen, R. K. Said, and U. S. Inan (2013), Statistical Patterns in the Location of Natural Lightning, J. Geophys. Res. Atmos., 118, doi:10.1002/jgrd.50107.

\section{Introduction}

[2] Lightning is one of the most powerful electrical processes on Earth, with billions of Joules of energy released in a typical flash [Rakov and Uman, 2007, pp.7]. Yet, the physical nature of lightning is complicated and not fully understood, in part due to the difficulty of making measurements inside thunderclouds, and in part due to the extremely wide range of timescales (from nanoseconds to hundreds of milliseconds) that characterize the various physical processes which occur.

[3] Lightning is formed inside cumulonimbus clouds, in particular those that feature strong convective updrafts that cause frozen water particles of different sizes and temperatures to rub and exchange charge, separating electrical charge vertically (citepMacGormanRust1998, ch.3). The classic picture of charge structure inside a thundercloud involves three layers of charge, a positive layer at the top, a negative layer in the middle, and a (sometimes

\footnotetext{
${ }^{1}$ Department of Electrical Engineering, Stanford University, Stanford, Calif., USA.

${ }^{2}$ Vaisala Inc., Boulder Operations, Louisville, Colo., USA.

${ }^{3}$ Electrical Engineering Department, Koc University, Sariyer, 34450, Istanbul, Turkey.

Corresponding author: F. G. Zoghzoghy, M. B. Cohen, R. K. Said, U. S. Inan, Department of Electrical Engineering, Stanford University, Stanford, CA, USA. (fadiz@stanford.edu)

(C)2013. American Geophysical Union. All Rights Reserved. 2169-897X/13/10.1002/jgrd.50107
}

ignored) smaller positive layer at the bottom [MacGorman and Rust, 1998, ch.3].

[4] Roughly $75 \%$ of lightning activity corresponds to intracloud (IC) discharges [Rakov and Uman, 2007, pp.4], which occur within the cloud and involve a breakdown connecting a positive and a negative layer. The remaining lightning activity is due to cloud-to-ground (CG) flashes, which transfer charge between cloud and ground. CG flashes are dangerous and can lead to fires, power outages, and casualties, mostly due to their high peak currents and their continuing currents that last tens of milliseconds and reach thousands of amperes [Rakov and Uman, 2007, pp.4]. Cloud-to-cloud and cloudto-air discharges are rare and often overlooked. Throughout our analysis we initially focus on CG flashes due to their practical applications and ease of detection, and then expand our analysis to IC flashes.

[5] Natural CG flashes either have a positive or a negative polarity and typically follow a downward propagating stepped leader, which is initiated by a preliminary breakdown within the cloud (still not a fully understood process). Negative CG (neg-CG) flashes are substantially more common, although the ratio varies by location [Orville et al., 2011. Positive CG (pos-CG) flashes initiate from a positive cloud layer, generally the top one, and usually consist of a single return stroke. The stroke is often followed by longenduring continuing currents, which reach tens of kiloamperes, approximately an order of magnitude more intense than neg-CG continuing currents [Rakov and Uman, 2007, pp.222]. Pos-CG flashes transfer a large amount of positive charge to ground, triggering upper atmospheric breakdown processes known as sprites [Cummer and Inan, 1997]. 
[6] The majority (90\%) of CG flashes are negative and initiate in the middle layer of the thundercloud [Jacobson and Krider, 1976]. Neg-CG flashes last for $\sim 1$ second and may consist of multiple (3-4 on average) return strokes separated by tens of milliseconds [Berger et al., 1975; Rakov and Uman, 2007, pp.4]. The first return stroke occurs in the ionized channel that was initially formed by the stepped leader. The following subsequent return strokes either recur along the same existing channel with a dart leader, or in a newly-formed channel up to several kilometers away from the first ground contact point with a dart-stepped leader [Rakov and Uman, 2007, pp.7]. The return stroke propagates upward along the ionized path at one-third the speed of light with typical peak currents of 20-30 kA.

[7] J- (for "Junction") and K- processes occur between successive return strokes (and can recur after the final return stroke) and transport charge from other regions of the cloud to the top of the ionized channel. The J-process lasts tens of milliseconds and carries charge horizontally toward the previous stroke, slowly re-building the local electric field. While the charge movement is toward the existing channel, subsequent strokes can still occur in other channels, redistributing charge in other parts of the storm. The K-process has much shorter timescales and is associated with abrupt jumps in the electric field with a risetime (10-90\%) shorter than $3 \mathrm{~ms}$ [Thottappillil et al., 1990; Rakov et al., 1992]. K-processes are sometimes interpreted as "attempted" leaders that propagate down the existing (but decaying) ionized path, which has an elevated temperature, but fail to reach ground and do not trigger return strokes [Rhodes and Krehbiel, 1989; Mazur et al., 1995].

[8] Lightning flashes occur in small spatial cells, over an area of $100-200 \mathrm{~km}^{2}$. These cells typically last on the order of an hour [Rakov and Uman, 2007, pp.25]. Flash rates from a cell are $\sim 1-5$ flashes per minute depending in part on the storm duration [Peckham et al., 1984]. Certain cells, known as supercells, can last substantially longer (several hours), while a class of storms known as mesoscale convective systems can cover exceptionally large areas [Rakov and Uman, 2007, ch.2]. These systems may consist of tens of thousands of CG lightning flashes, clustered into many cells. Various studies show that, during peak lightning activity or in severe weather conditions, the ground flash rates could be an order of magnitude higher, exceeding 20 flashes per minutes [Williams, 1985; Rakov and Uman, 2007, pp.25].

[9] Flashes neutralize a fraction of the charge separation within the cloud leading to sharp variations in the ambient electric field (We note that, throughout the paper, we use the terms "neutralize" and "destroy" to describe, in the macroscopic sense, the charge redistribution process that results from lightning and not to suggest that charge is microscopically neutralized). These variations and the subsequent electric field recovery curves were first measured on the ground below thunderstorms in the 1910s and were used to study the electrification processes and the charge structure inside thunderclouds [Wilson, 1916]. Following a lightning discharge, the electric field at the Earth's surface takes tens of seconds to get back to its pre-flash value, with fast initial recovery rates that decrease over time [Wilson, 1916; Wormell, 1939; Gopalakrishnan et al., 2011]. The recovery rates are also proportional to the cloud convective activity, with faster recoveries for stronger activity levels [Jacobson and Krider, 1976]. These ground measurements provide accurate timescales for charge buildup inside thunderstorms but the shapes of these ground-level electric field recoveries could be affected by point discharge from the ground or by the finite conductivity of air outside the cloud [Illingworth and Wormell, 1971; Chalmers, 1967].

[10] To further study the electrification processes of thunderstorms, balloon-borne instruments with electric field sensors have been used to monitor the impact of a lightning discharge on the electric field inside the storm and to extract the true shape of the subsequent recovery. For instance, Winn and Byerley, [1975] monitored the variations of the magnitude of the electric field inside storms following forty-four lightning flashes and found that on average the magnitude of the electric field dropped by $40 \%(20 \%$ standard deviation) and recovered linearly. Such field experiments have quantified the impact of lightning and have modeled the amount of charge lowered to ground, but these studies collected limited amount of data around the experimental setup. The aim of our work is expand these studies to a global scale and to monitor the charging and discharging processes of storms using lightning geo-location data and statistical techniques that bring out these recovery patterns. This could potentially allow the analysis of the electric field recoveries for various lightning parameters, storm types, geographical regions, and seasons.

[11] Dennis [1970] presents the delay times between flashes in a storm, with visual and radar observations, and finds a very small autocorrelation, indicating a nearly independent process. This early study of the temporal pattern of lightning flashes in a given storm cell suggests that the flashing process of a storm is essentially time-independent, and that it could thus be modeled as a Poisson random process. This hypothesis implies either that the spatial scales within a thundercloud are very small [Kuettner, 1950], or that the charge neutralized in a flash is relatively small compared to the amount that was present before the flash, leaving the basic cloud charge structure largely unchanged as a result of any given flash, or that the charge separation rebuilds extremely quickly. But recent studies have agreed that the successive flashes in a storm cannot be modeled as an independent process, implying that a typical lightning flash destroys a substantial fraction of the cloud charges (requiring a recovery time before the next flash). Finke [1998] used lightning geo-location data as a proxy measure of thunderstorm characteristics, analyzing the autocorrelation function of the lightning distribution and empirically deriving events such as velocity, lifetime, and size of lightning storms (averaged over a regional storm). While focusing on longer term (many minutes) effects related to cell evolution and motion, Finke [1998] briefly mentions the oscillatory nature of lightning within a storm cell, in which a lightning flash suppresses the probability of another flash until enough time has passed for the electrical conditions allowing a flash to be re-established.

[12] In addition, there is some evidence that spatially separated cells in a thunderstorm system may be partially coupled, leading to synchronized behavior between them, for reasons not yet understood. This synchronized behavior was first observed from space and later Mazur [1982] established this phenomenon using VHF radar reflections from a thunderstorm. Vonnegut et al. [1985] provided anecdotal evidence of multi-cell coupling using video recordings from STS missions. Yair et al. [2006] and Yair et al. [2009] 
provided some quantitative evidence of this coupling effect using recordings from the ill-fated STS-107 Columbia mission, and from ground lightning networks, respectively, explaining the results in terms of networked coupled oscillators.

[13] In this paper, we present a statistical technique similar to that of Finke [1998], but applied to the shorter lengths and timescales characteristic of the charging and discharging processes in a storm, and using more lightning data with more accurate geo-location accuracy and detection efficiency (discussed in the next section). We propose that this technique could be used as a proxy measure of the characteristic charging time and a measure of the percentage of charge transferred in a lightning flash, and could also be used to characterize the synchronicity of lightning cells. This statistical tool can be used as a surrogate measure of electric field recovery and can be applied on a global level for thousands of storms to study the effects of seasonality, geographic location, and weather parameters on lightning. These advances may contribute both to lightning protection and prediction systems.

\section{Observations}

\subsection{Description of Data}

[14] We utilize lightning geo-location data both from the National Lightning Detection Network (NLDN) and the GLD360 Network.

[15] NLDN started in the 1980s as a research network at SUNY/A. The network expanded to cover the contiguous U. S. by 1989 with the support from the Electric Power Research Institute (EPRI). Later, Global Atmospherics operated NLDN until 2002, when the company was sold to Vaisala Inc. NLDN is comprised of $\sim 150$ sensors across the continental USA, detecting the Very Low Frequency (VLF; 3-30 kHz) and Low Frequency (LF; 30-300 kHz) radio emissions from lightning. The detection efficiency is estimated to be $\sim 60-80 \%$ for cloud-to-ground (CG) strokes [Cummins and Murphy, 2009] and the polarity and a model-derived measure of peak current of each event are reported. These model-derived peak current measurements have been tested for neg-CG subsequent return strokes in triggered lightning [Cummins and Murphy, 2009]. NLDN also detects a small number $(\sim 10-20 \%)$ of intracloud (IC) flashes. The NLDN geo-location accuracy is $\sim 400 \mathrm{~m}$ with a median error of $308 \mathrm{~m}$ [Nag et al., 2011]. The efficient and accurate detection is possible because NLDN sensors are close enough to the source to detect the ground wave. Said et al. [2010] developed techniques to extend this high detection efficiency on a global scale, achieving 70\% CG flash detection efficiency and 2-5 km accuracy using the efficiently propagating subionospheric VLF component, in the GLD360 network. GLD360 is a long-range lightning geolocation network that was jointly developed by Stanford University and Vaisala Inc., and has been operating continuously since 2009. It employs a global network of VLF sensors that use magnetic direction finding and time-of-arrival methodologies to detect and locate individual lightning discharges around the world. Using a propagation correction model, the network uses the magnetic field intensity measured at each sensor to estimate the peak current of each detected event [Said et al., 2010]. First, we analyze NLDN data from the continental USA and then expand our analysis to study oceanic lightning using GLD360 data.

\subsection{Definition of a Lightning Cluster}

[16] We pre-process the lightning data by grouping neighboring lightning events into clusters of lightning activity. A given lightning discharge $i$ belongs to cluster $C_{n}$ if it occurs within 10 minutes and 60 kilometers from a previous discharge $j$ that already belongs to $C_{n}$. This technique groups neighboring lightning activity and storm cells into large lightning systems, which we define here as lightning clusters. For instance, we first apply this clustering approach to NLDN neg-CG return stroke data from a particular day (August 23, 2007) with strong lightning activity. Figure 1 shows a map of the four largest lightning clusters which occurred that day. There were a number of other lightning clusters, but only the four largest (by number of CG return strokes) are shown, each lasting several hours and consisting of squall line mesoscale convective systems. The sole purpose of this clustering is to group lightning events, storm cells, and storms into a single lightning cluster. Then we analyze that cluster, potentially looking for interactions between nearby storm cells (as discussed earlier). Hence, the chosen clustering parameters (60 km, $10 \mathrm{~min})$ could be varied but should be large enough to include all the neighboring activity.

[17] Next, we use statistics to extract the average impact of a lightning event on the activity within its lightning cluster. Throughout our analysis, we assume that this impact is stationary and does not vary with time, geographic location, and other characteristics of the corresponding lightning cluster (such as activity level, duration, and phase). The analysis of the impact of lightning for different storm types and phases requires complementing the global lightning geo-location data with global radar data, but such global radar data are unavailable. This could be the subject of a future study where the analyzed lightning data correspond to a specific region with available radar data.

\subsection{Statistical Approach}

[18] The aim is to quantify the impact of a lightning event on the surrounding lightning activity, assuming that this effect is stationary. To do so, we use the following approach: around each lightning event in space and time, other neighboring events will occur based on the probability of an event occurring at that location and time delay. For instance, for a lightning event $i$ occurring at time $t_{i}$, latitude $\lambda_{i}$, and longitude $\phi_{i}$ (hence position $\left(\lambda_{i}, \phi_{i}\right)$ ), another event $j$ in the same lightning cluster $C_{n}$ can be written by its relative time of occurrence and position compared to $i$, as shown in Equation 1. Throughout the text, we refer to event $i$ as the reference event.

$$
\left(\Delta t_{i j}=t_{j}-t_{i}, \Delta d_{i j}=\text { distance from event } i \text { to } j\right)
$$

[19] This process is then repeated for all the events of $C_{n}$ and the results are summed up to construct a two-dimensional lightning-occurrence histogram of the relative distances and time delays of the events in $C_{n}$. This histogram can be thought of as the autocorrelation function of the lightning activity [Finke, 1998]. The occurrence histogram could be used to either monitor the lightning activity in a given region as a function of time delay (fix the value of $\Delta d$ and vary $\Delta t$ ) or to monitor the spatial distribution of the lightning activity for a given time delay (fix the value of $\Delta t$ and vary $\Delta d$ ). The results are valid for relative times and locations that are respectively 


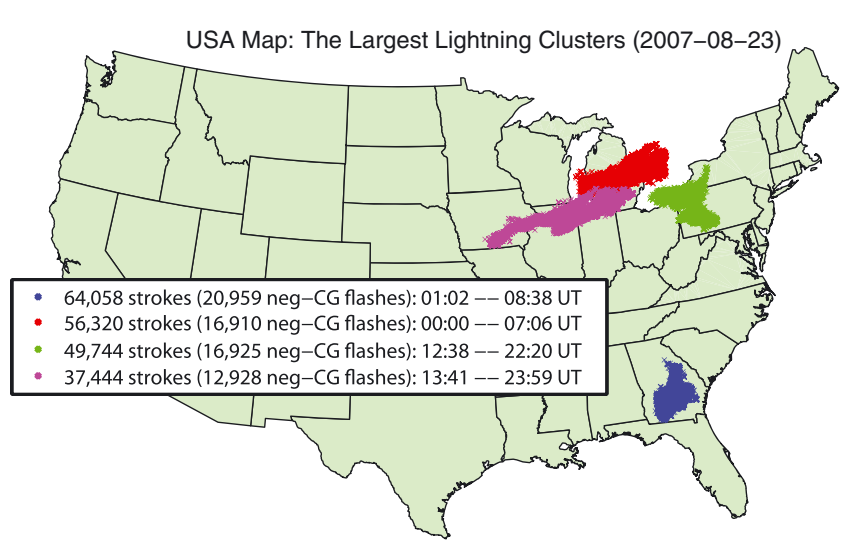

Figure 1. Lightning activity from NLDN over the continental USA, on 23-Aug-2007. Only the four largest lightning clusters are shown, with some tens of thousands of strokes over several hours. The number of flashes were determined using a stroke-to-flash clustering algorithm [Cummins et al., 1998].

less than 10 minutes and 60 kilometers, which are the parameters used for the grouping of the lightning clusters.

[20] It can be presumed that for large time delays, $\Delta \mathrm{t}>\Delta t_{\max }$, or for large distances, $\Delta \mathrm{d}>\Delta d_{\max }$, the number of events in the histogram should be roughly constant (as a function of time $\Delta t$ ). Such a result is expected because a lightning discharge's impact on its surroundings extends only over a finite distance and time, which we define respectively as $\Delta d_{\max }$ and $\Delta t_{\max }$. At a great enough time, or space separation, two chosen lightning flashes are independent; thus, the probability of an event at distance $\Delta d$ and time delay $\Delta t, p(\Delta d, \Delta t)$, is constant as a function of time if one of the aforementioned conditions is satisfied. Hence, if we repeat the analysis around $\mathrm{N}$ reference events, the steadystate values will be roughly equal to $N^{*} p(\Delta d, \Delta t)$. Throughout our analyses, we extract the values of $\Delta d_{\max }$ and $\Delta t_{\text {max }}$ (roughly the spatial and temporal extent of the impact of lightning) for different lightning parameters.

\subsection{Application to Neg-CG Stroke Data}

[21] First, we apply this technique to NLDN neg-CG return stroke data, for only the largest lightning cluster shown in blue in Figure 1. There were a total of 64,058 strokes in this lightning cluster over Georgia. The histogram is constructed after summing up all the relative distances and time delays of all 64,058 strokes in this cluster. Figure 2 shows the relative occurrences of lightning strokes as a function of distance and parameterized by time delay, showing the spatial distribution of neg-CG return strokes around other neg-CG return strokes for four different time delays (one second long windows). The curves are parameterized by the first, second, third, and fifteenth seconds after the reference lightning stroke. The relative locations of the strokes, $\Delta d$, are binned in $250 \mathrm{~m}$ distances. The four time windows are selected (a) because the first three display the lightning activity during and right after the CG discharge and (b) because the last time window $\left(15^{\text {th }}\right.$ second) corresponds to ground-truth timescales for electric field recovery inside storms [Wilson, 1916]. In this example, only negCG lightning with NLDN-recorded peak current $>5 \mathrm{kA}$ and reasonably small location uncertainty (with chi-squared value

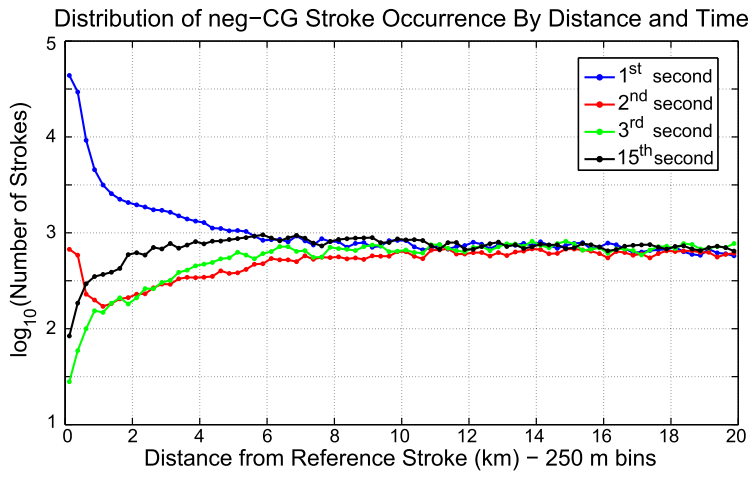

Figure 2. The number of strokes as a function of distance from the source stroke, plotted separately for the $1^{\text {st }}$ second (blue), $2^{\text {nd }}$ second (green), $3^{\text {rd }}$ second (red), and $15^{\text {th }}$ second (black). NLDN Data from the largest lightning cluster of 23-August-2007 are used.

of the geolocation optimization algorithm smaller or equal to 2) are included.

[22] Neg-CG strokes occurring within one second are dominated by those that are part of the same flash, since a flash typically lasts on the order of a second. For this particular lightning cluster, within the first second there are three notable components (blue curve): (1) A very large number of strokes values in each bin occurring in nearly the same location (i.e., within the $\sim 400 \mathrm{~m}$ NLDN geo-location uncertainty). (2) An elevated number of strokes within $\sim 5 \mathrm{~km}$, corresponding to strokes that are part of the same lightning flash but with different channels or ground contact points. This $5 \mathrm{~km}$ distance corresponds roughly to the horizontal extent of the average charge redistribution in the cloud, for this particular cluster. The more modestly elevated number of strokes between $5-10 \mathrm{~km}$ may be due to a small number of particularly large cells, or may also be due to a coupling mechanism between distinct cells as discussed by Yair et al. [2006]. (3) An independent region $>10 \mathrm{~km}$ where the number of strokes occurring roughly reflects the expected number given the overall stroke rate of the cluster.

[23] The curve for the $2^{\text {nd }}$ second following a stroke (red curve) is dominated by subsequent strokes that are near the end of the flashes, with an elevated number of strokes along the original channel $(0-1 \mathrm{~km})$ and a substantially smaller number of subsequent strokes in new contact points $(\sim 1-5 \mathrm{~km})$. This result is consistent with past observations that strokes in the later portion of the flash are more likely to occur along an existing channel than along a newly formed dart-stepped leader channel [Stall et al., 2009]. Thus the first two seconds following a neg-CG return strokes are dominated by subsequent strokes that occur part of the same flash. This suggests that a neg-CG lightning discharge could last longer than one second, consistent with previous field observations [Berger et al., 1975; Saraiva et al., 2010]. The independent region $>10 \mathrm{~km}$, described in the previous paragraph, remains unchanged.

[24] In the $3^{\text {rd }}$ second following a stroke (green curve), the number of strokes occurring near the reference stroke $(0-1 \mathrm{~km})$ drops to a minimum, the original flash is nearly always extinguished, and a new dynamic is apparent. For distances below $10 \mathrm{~km}$, there is a clear suppression in the number of strokes, as new flashes are less likely to be 
established near the previous flash. The effect is more prominent closer to the location of the discharge and recovers with time. This can be seen in the curve corresponding to the $15^{\text {th }}$ second which shows an increase in the number of strokes occurring around the location of the initial stroke, compared to the number of strokes in these location occurring during the $3^{\text {rd }}$ second (recovery). This implies that the lightning flash partially destroys the background conditions for a flash. The lack of any suppression (or enhancement) in the number of strokes beyond $12-20 \mathrm{~km}$ (the number of strokes in these regions is the same for the four different time windows) would seem to be an indication that (1) the impact of lightning only extends over a finite distance, for this particular cluster $\Delta d_{\text {max }}=$ $12 \mathrm{~km}$ and that (2) the coupling mechanism between storm cells described by Yair et al. [2006] and Yair et al. [2009] is not occurring in this particular lightning cluster, although it is possible that this effect is either too small to be detected, or is not brought out with this statistical technique.

[25] The background conditions that lead to lightning are still not fully understood; however, it is generally agreed that an intensification in the local electric field causes the dielectric breakdown of air, eventually leading to a lightning flash [Hagenguth, 1951]. Thus, the probability of a dielectric breakdown, hence lightning, is correlated with the electrical conditions inside the cloud: the probability of a lightning flash in a given region of the cloud is proportional to the strength of the local electric field in that region. Consequently, monitoring the recovery of the probability of a lightning flash at a given distance $\Delta d$ from the previous flash and as a function of time delay $(\Delta t)$ can provide a surrogate measure of the recovery of the electric field at that relative distance $\Delta d$ from the discharge. We follow this approach to characterize the suppression effect that is shown in Figure 2, extracting the timescales and the shapes of the recoveries, and quantifying the temporal and spatial extents of a lightning event (respectively $\Delta t_{\text {max }}$ and $\Delta d_{\max }$; defined previously). In the following section, we use lightning flash data to quantify this flash suppression effect.

\subsection{Application to Neg-CG Flash Data}

[26] We follow the same statistical approach to analyze NLDN neg-CG flash data (instead of stroke data). The NLDN neg-CG stroke data are clustered into neg-CG flash data using a clustering algorithm similar to the one described by Cummins et al., [1998]. The clustering tolerances used here for a neg-CG flash are $\sim 1$ second and a maximum radius of 10 kilometers. We produce the two-dimensional occurrence histogram using all the neg-CG flashes from August 23, 2007 (274,860 neg-CG flashes). Figure 3 shows the recovery in different regions around the neg-CG lightning flash as a function of time. The area around the discharge is divided into concentric rings of $2 \mathrm{~km}$ thickness and the time delays are sorted into 1 second bins. For visualization purposes, each curve is normalized to plateau at a value of 1 . This is done by dividing the entries of a given row of the occurrence histogram (for a given $\Delta d$ ) by the steady-state (post-recovery) flashing rate, which is reached at $\Delta t_{\max }$. For instance, the blue curve shows the recovery of the probability of a subsequent neg-CG flash in the $0-2 \mathrm{~km}$ region as a function of time. We note that the first two seconds in the plot are ignored because they correspond to the original flash duration, as discussed in the paragraph following Figure 2.

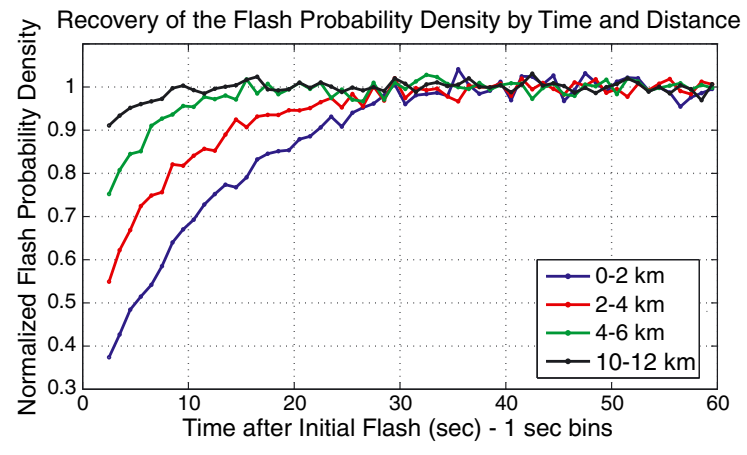

Figure 3. The flash probability density as a function of time, separately parameterized by concentric rings from the source. The recoveries in the $0-2 \mathrm{~km}$ region (blue), $2-4 \mathrm{~km}$ region (red), 6-8 $\mathrm{km}$ region (green), and 10-12 $\mathrm{km}$ region (black) are shown. All neg-CG flash data from August 23, 2007 are used.

[27] The recovery time $\Delta t_{\max }$ varies for different regions around the discharge, with the region nearest to the flash (blue curve) recovering in $\sim 30$ seconds, the region around $5 \mathrm{~km}$ (green curve) recovering in only $\sim 15$ seconds, and the farthest region of the cloud (black curve) recovering in only 5 seconds. Similarly, the magnitude of the suppression is more pronounced near the source discharge (blue curve), with a $\sim 62 \%$ drop in post-flash lightning activity, while at more distant regions (red, green, black curves) the activity only drops by $45 \%, 25 \%$, and $9 \%$ respectively. Hence, the probability suppression effect closes in as time advances, eventually lingering only very close to the original neg-CG flash. This indicates that, at least for this particular summer day, the charge redistribution at 5-10 km horizontal distance from the flash is present, but the effect is much stronger at the flash location. The results depicted in Figure 3 are consistent with timescales of electric field recovery measured in field experiments [Wilson, 1916; Wormell, 1939; Jacobson and Krider, 1976]. The magnitude of the drop in the flashing probability around the flash vary betweeen $25 \%$ and $62 \%$ which is also consistent with balloon-borne experiments that measure the drop in the magnitude of the electric field inside the storm following a lightning discharge [Winn and Byerley, 1975]. These similarities between the electric field recovery and the flash probability recovery indicate that the proposed method is a valid surrogate measure of electric field buildups in thunderclouds and could be applied to study thunderstorm electrification processes for different lightning parameters for years of available lightning data, for which consistent and global electric field measurements or radar data are unavailable.

\subsection{Neg-CG Multiplicity}

[28] Next, we study the probability suppression effect as a function of the multiplicity of the neg-CG discharge (the number of return strokes per flash). Figure 4 illustrates the suppression effect for NLDN neg-CG lightning as a function of neg-CG flash multiplicity. The same statistical technique is applied to all the lightning activity from August 22/23/ 24,2007 but is done separately to study the lightning activity around multiplicity 1 reference flashes (single return stroke flashes) (blue curve), multiplicity 2 reference flashes 


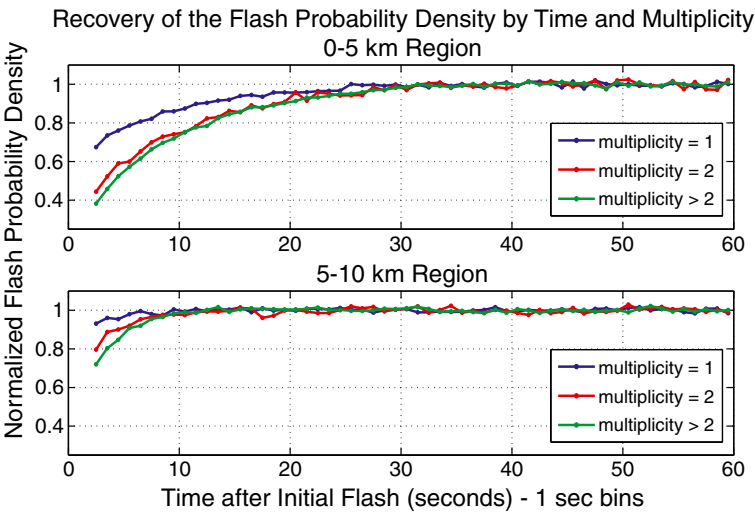

Figure 4. (Top) The flash probability density in the $0-5 \mathrm{~km}$ region around the source discharge as a function of time, parameterized by flash multiplicity. The flash probability densities are plotted separately for flashes with multiplicity 1 (blue), multiplicity 2 (red), and higher multiplicities (green). (Bottom) Similar analysis but for the $5-10 \mathrm{~km}$ region. Neg-CG flash data from August 22/23/24, 2007 are used.

(red curve), and for the remaining flashes with higher multiplicities (green curve), with the following neg-CG flashes being of any multiplicity. The multiplicities are computed after clustering NLDN neg-CG strokes into flashes. Due to the lower NLDN detection efficiency for weaker subsequent return strokes [Nag et al., 2011], the resulting NLDN multiplicities are lower than the true flash multiplicities, but the two measures are highly correlated. The curves are then normalized by their corresponding post-recovery values to plateau at a value of 1 . We monitor the recovery in two different regions: one near the source discharge $(0-5 \mathrm{~km}$; top panel), and one in a more distant region $(5-10 \mathrm{~km}$; bottom panel), as a function of time delay (1 second bins). The first two seconds in the plot are ignored because they correspond to the original flash duration (discussed earlier).

[29] We find that, on average, higher multiplicity flashes lead to longer and stronger probability suppression effects. In the region near the flash (top), the probability of a subsequent flash recovers in only $\sim 25$ seconds following a single-stroke flash (blue curve) while the recovery takes $\sim$ 30 seconds after multiple-stroke flashes (red, green curves). In addition, the flash occurrence rate only drops by $\sim 32 \%$ in the single-stroke case (blue curve), while the other two curves (red, green curves) drop $\sim 56 \%$ and $\sim 62 \%$ respectively. The more pronounced suppression effect following higher multiplicity flashes indicates that the total charge neutralized by a flash is proportional to the flash multiplicity; additional return strokes transport more charge to ground, requiring a longer re-charging process before another flash can take place.

[30] The curves corresponding to the single-stroke flashes (blue curves, top and bottom panels) show a substantial suppression effect in the region nearest to the flash (top) but a substantially smaller effect in the $5-10 \mathrm{~km}$ region (bottom), where the flash probability is uniform over time. This indicates that a single return stroke mostly neutralizes charge from the region around the stroke and does not destroy an appreciable portion of the charge from more distant regions $(5-10 \mathrm{~km})$, which seems to suggest that a single return stroke, on average, has a maximum spatial extent of 5 kilometers.
[31] On the other hand, the two curves corresponding to multiple-stroke flashes (red and green curves) still show a significant suppression effect at $5-10 \mathrm{~km}$. This suggests that multiple-stroke flashes can neutralize charge from distant locations of the cloud, more than 5 kilometers away from the initial channel. These conclusions are consistent with results from field experiments where an electric field measuring system is used to study the charge source locations for subsequent return strokes and continuing currents in multiple-stroke flashes in New Mexico [Krehbiel et al., 1979]. We thus note a clear difference in the nature of the first and subsequent strokes, as is apparent in the flash occurrence patterns. The more distant reach of the multiple-stroke flashes may be due to either subsequent return strokes occurring in a newly formed channel (a few kms away) following a dartstepped leader, or to J- and K- processes which transport charge from other regions of the cloud to the top of the existing channel before the subsequent return stroke.

\subsection{Neg-CG Peak Current}

[32] Figure 5 shows the flash suppression effect as a function of NLDN-reported return stroke peak current, determined separately for intense (>30 kA, in red) or less intense (5-20 kA, in blue) neg-CG strokes from August 22/23/24, 2007. Only single-stroke flashes are analyzed to eliminate the effect of multiplicity. More intense lightning return strokes seem to more strongly suppress the probability of subsequent flashes, but the recovery characteristics appear to be similar. This may indicate that more intense neg-CG strokes are redistributing a larger amount of charge, but are also occurring in systems where the charging currents are particularly strong, so that recovery from this suppression is faster so as to counteract the larger charge neutralization. Although the peak current of the return stroke is not necessarily proportional to the total charge transfer in the lightning stroke (since the duration of the stroke and the intensity of continuing currents is important), since we are considering here only a specific type of stroke (neg-CG) they are at least correlated.

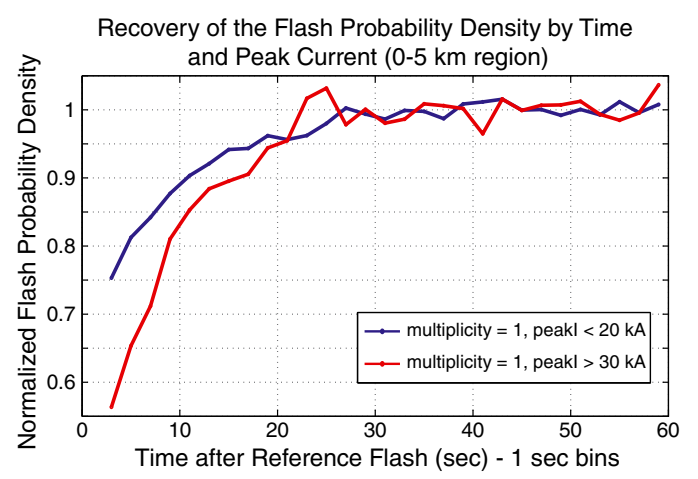

Figure 5. The flash suppression effect as a function of time and parameterized by distance, separately for neg-CG singlestroke flashes with peak currents in the 5-20 kA range (blue) and for neg-CG single-stroke flashes with peak currents greater than $30 \mathrm{kA}$. Single-stroke neg-CG flash data from August 22/23/24, 2007 are used. 


\section{Discussion}

[33] In the previous section, we introduce our statistical approach and use it to study the flash suppression effect for neg-CG flashes of different multiplicities and intensities. In this section, we expand our analysis to compare the suppression effect of oceanic and land lightning and to study interactions between flashes with different polarities and types (cloud and ground flashes). Such studies show the various applications of this statistical technique and provide more insight about lightning (which is hard to observe otherwise).

\subsection{Flash Polarity}

[34] The previous results show that neg-CG flashes affect the probability of subsequent neg-CG flashes, implying destruction of charge in the negative cloud charge layer. Figure 6 shows how the flash suppression effect varies with different polarities. The flash probability recovery as a function of time delay from the originating flash is shown, encompassing all flashes within $5 \mathrm{~km}$ of the original. The flash suppression observed in red in Figure 6 concerns neg-CG flashes following other neg-CG flashes, and is similar to the effect previously shown in Figure 3 but is shown here for a longer duration (10 minutes). This curve is calculated from all neg-CG flashes following (within 10 minutes and 5 kilometers) 274,860 reference neg-CG flashes, occurring on August 23, 2007, as detected by NLDN. The plot includes a logarithmic horizontal axis to show a large range of timescales, and is normalized by the steady-state flashing rate.

[35] The same curve derived using positive polarity CG (pos-CG) flashes following other pos-CG flashes is shown in blue. The curve for neg-CG flashes following pos-CG flashes is shown in green; here we compute the occurrence histogram by summing up the relative time delays and distances of neg-CG that follow pos-CG flashes (within 10 minutes and 5 kilometers). Since there are fewer positive flashes in general, to form enough statistics for the cross polarity events and for the positive polarity analysis (green and blue curves), all NLDN data from August 22/23/24, 2007 were combined, yielding 57,098 reference pos-CG flashes. For this analysis, only pos-CG strokes stronger than

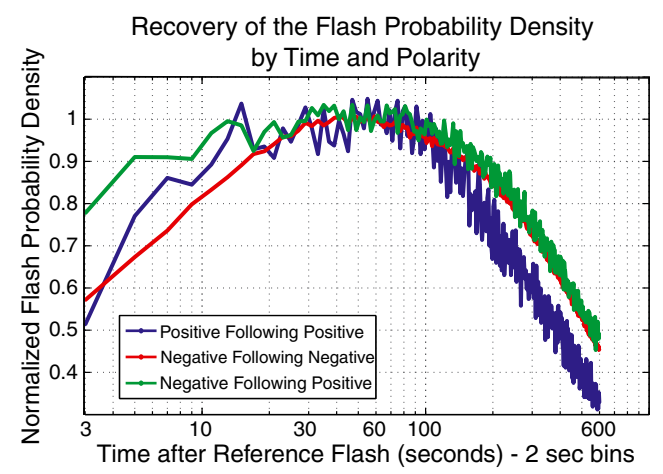

Figure 6. The flash suppression effect as a function of distance and parameterized by flash polarity in the $0-5 \mathrm{~km}$ region. The recovery of pos-CG following pos-CG (blue), neg-CG following neg-CG (red), and neg-CG following pos-CG are shown. CG flash data from August 22/23/24, 2007 are used.
$15 \mathrm{kA}$ are used, for which the source type and polarity determination by NLDN is more certain [Nag et al., 2011].

[36] There does appear to be a corresponding positive flash suppression effect (blue curve), similar in intensity and duration to the negative flash suppression effect, as seen by the rise in probability density over the first $\sim 30$ seconds after the flash. There is also a detectable, though weaker, cross polarity effect, in which positive flashes suppress subsequent negative flashes. Hence, it would appear that on average, pos-CG flashes (1) may also be draining at least some charge from the negative region in a cloud, or (2) may be reducing the electric field between the negative and positive regions which subsequently reduces the probability of a negative discharge. Unfortunately, there were not enough statistics on these days to determine the flash suppression effect for positive flashes following negative flashes.

[37] Additionally, the effects of storm motion can be seen over a long time scale. After a couple minutes time, all three curves show a steady decrease in the flash probability with time. This occurs when the storm center has moved far enough away that the $5 \mathrm{~km}$ circle no longer includes the storm center. The general trend of the decrease (in lightning probability) with storm motion is the same for the red and green traces, but different for the positive-polarity distribution, shown in blue. This may be due to the fact that positive lightning more often occurs in different parts of the storm, for instance, in the trailing portions of mesoscale convective complexes where the negative layer may have been partially depleted by repetition of neg-CG discharges [Lyons, 1996]. So the physical area over which a positive lightning flash is likely to occur may be different from that of a negative flash, so that the downward trend from storm motion has a different characteristic. The interpretations of these trends could be further explored using radar data [Williams and Yair, 2006].

\subsection{Ground and Cloud Flash Type}

[38] So far, we have focused on CG land lightning flashes. In Figures 2-5 we work with neg-CG data and in Figure 6 we study the interactions between negative and positive polarity CG flashes. In this subsection, we explore the flash probability suppression effect for different flash types (IC and CG) using NLDN IC and CG data. We note that our statistical technique is not affected by the low IC NLDN detection efficiency $(10-20 \%)$. The low detection efficiency is equivalent to a random sampling of the entries of the occurrence histogram to remove data points, deleting equal number of samples from each histogram entry (assuming that the detection efficiency is uniform for IC flashes). This process does not affect the underlying probability distribution but requires a larger sample size (more lightning data) to better estimate the recovery patterns. Here, we use all lightning data collected by NLDN from 02-25 July, 2011.

[39] Figure 7 presents the interactions between different flash types. The first panel (top-left) shows the distribution of $\mathrm{CG}$ flashes (both polarities) following a $\mathrm{CG}$ flash, the second (top-right) shows the distribution of IC flashes (both polarities) following an IC flash, the third (bottom-left) is for IC activity following a CG discharge, and the fourth (bottom-right) is for CG activity following an IC discharge. Each panel is produced using the aforementioned statistical method applied to all NLDN IC and CG data from 02-25 July, 2011 (6,271,850 IC flashes, 13,653,455 CG flashes). 


\section{ZOGHZOGHY ET AL.: LIGHTNING FLASH SUPPRESSION}

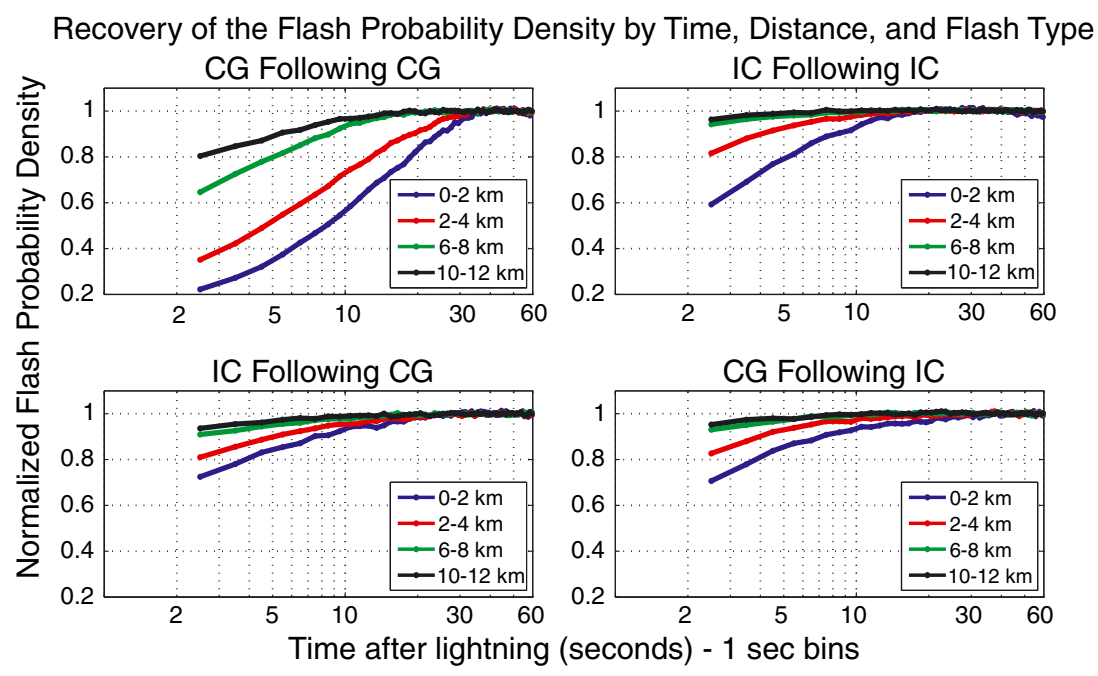

Figure 7. The flash suppression effect as a function of time parameterized by flash type and distance from the discharge: (top-left) recovery of CG lightning probability following a CG discharge, (top-right) recovery of IC lightning probability following an IC discharge, (bottom-left) recovery of IC lightning probability following a CG discharge, (bottom-right) recovery of CG lightning probability following an IC discharge. The recoveries in the 0-2 $\mathrm{km}$ region (blue), $2-4 \mathrm{~km}$ region (red), 6-8 $\mathrm{km}$ region (green), and 10-12 km region (black) are shown. NLDN CG and IC flash data from July 02-25, 2011 are used.

For instance, the occurrence histogram of the third panel is generated by counting the relative distances and time delays of IC flashes that follow CG flashes. The plots include a logarithmic time axis and are normalized by the steady-state flashing rate (plateau at a value of 1). The first two seconds are ignored (discussed earlier).

[40] All four panels suggest that the flash probability suppression effect exists for the four scenarios, but the strength of the suppression, in terms of duration and magnitude, varies. In the 0-4 km region (blue and red curves; panel 1-4), the lightning activity following any type of discharge is affected for tens of seconds, implying that a lightning flash partially destroys the local conditions for a subsequent discharge. The more prominent suppression effect corresponds to the CG following CG case (Panel 1). The impact of a CG flash (on other CG lightning) extends to $\sim 12$ kilometers $\left(\Delta d_{\max }\right)$ and affects the region around the CG flash for $\sim 30$ seconds (top-left panel, in blue). The magnitude of the drop in lightning probability varies from $\sim 76 \%(0-2 \mathrm{~km}$, in blue $)$ to $\sim 37 \%$ in more distant regions $(6-8 \mathrm{~km}$, in green).

[41] The second panel of Figure 7 shows the probability distribution of IC flashes following IC flashes. The recovery pattern is still present but is much weaker than the CG following CG case (Panel 1). The impact here extends to only $\sim 5$ kilometers $\left(\Delta d_{\max }\right)$ and affects the region around the $\mathrm{CG}$ flash for $\sim 15$ seconds (top-right panel, in blue). The magnitude of the drop in lightning probability varies from $\sim 40 \%$ $(0-2 \mathrm{~km}$, in blue) to $<5 \%$ in more distant regions $(6-8 \mathrm{~km}$, in green). Although IC lightning may or may not neutralize more charge than CG lightning, our results suggest that an IC discharge does not affect the conditions for another IC discharge as efficiently as a CG discharge affects the conditions for another $\mathrm{CG}$ discharge. This suggests a fundamental difference between IC and CG flashes and their corresponding initiation and electrification processes.

[42] The third panel (bottom-left) and the fourth panel (bottom-right) present the probability distribution for IC following $\mathrm{CG}$ and for CG following IC respectively. Both recoveries only extend to $\sim 4 \mathrm{~km}$ and are similar in magnitude and in duration. The resemblance between the cross-type results indicate that both lightning types equally affect the flashing conditions of the other. The cross suppression effects are present but not as strong as the same-type suppression effects (Panel 1 and 2), suggesting that a lightning flash is more effective in destroying the conditions of a subsequent flash of the same type. Figure 7 provides more insight into the different types of discharges and the physics behind lightning, but the theoretical interpretations of these results are beyond the scope of this paper.

\subsection{Land and Ocean Lightning}

[43] Although lightning occurs about an order of magnitude more frequently over land, recent studies have shown that the average measured fields radiated from oceanic lightning is much higher [Orville et al., 2001; Orville et al., 2011; Lyons et al., 1998; Cummins et al., 2005; Said et al., 2013]. This indicates that either the meteorology of oceanic lightning, or the effect of highly conductive seawater, has a remarkable tendency to intensify the observed field when an oceanic flash does occur. It is still unclear if this observed enhancement is due to an increase in the average peak current or due to the attachment mechanism over saltwater.

[44] Figure 8 compares the recoveries following land lightning (solid-line curves) to the recoveries following oceanic lightning (dashed-line curves), parameterized by distance from the lightning discharge. The plots are produced using the same statistical approach applied to all GLD360 flash data from 02-25 July, 2011. GLD360 lightning data are global and include both IC and CG discharges (GLD360 does not classify the flashes). The first two seconds are ignored (discussed earlier) and the curves are normalized by their steady-state flashing rate (plateau at a value of 1). 


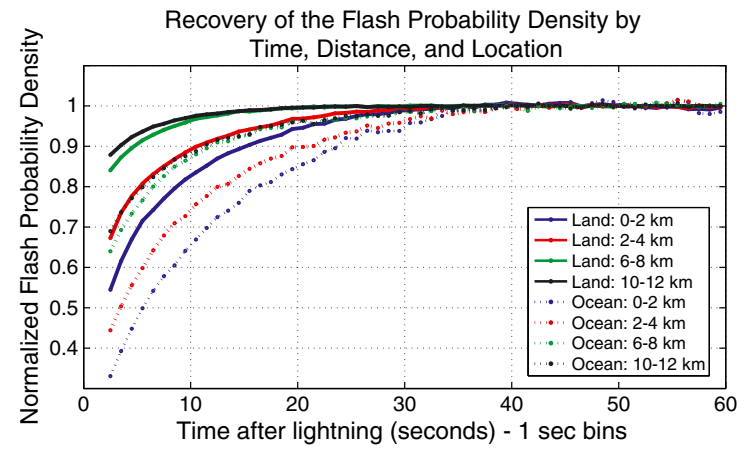

Figure 8. The flash suppression effect as a function of time for land and for oceanic lightning parameterized by distance. The solid lines correspond to the recovery following land lightning and the dashed lines for the oceanic lightning recovery. All GLD360 flashes from July 02-25, 2011 are used

[45] The results suggest that more prominent suppression effects follow oceanic lightning. The lightning activity in the region around the oceanic discharge $(0-2 \mathrm{~km}$, dashedblue) drops by $\sim 65 \%$ and recovers in $\sim 40$ seconds. The activity in the same region around a land discharge $(0-2 \mathrm{~km}$, solid-blue) only drops by $\sim 45 \%$ and recovers in only $\sim 30$ seconds. The remaining curves show that the average impact of land lightning on the $2-4 \mathrm{~km}$ region (solid-red) is comparable to the impact of an oceanic discharge on the more distant 10-12 km region (dashed-black), providing a rough idea about the greater extent and duration of the post-oceanic lightning suppression effect. These results suggest either that (1) oceanic lightning destroys more charge due to its high intensity [Said et al., manuscript in review, 2013], leading to longer recoveries, or that (2) oceanic storms have slower charging processes (weaker convective activity) than land storms, hence take more time to recover.

\section{Conclusion}

[46] In this paper, we have introduced a method of monitoring the impact of lightning and the charging and discharging processes of thunderstorms using large amounts of accurate geo-location data. The storm parameters described here are essentially averaged over a collection of storms, which consist of many different appearing and disappearing storm cells. It is very possible that different cells have different characteristics. The data here can be subdivided into smaller pieces to characterize these variations, up to the point where there are not enough statistics to observe the necessary parameters. We have not presented here variations of these parameters across different storm cell activity level, duration, season, etc. (using radar data). These studies are beyond the scope of the present paper.

[47] The suppression effect of subsequent lightning flashes implies that the flash neutralizes a substantial fraction of the charge built up in the cloud, affecting the background conditions of a subsequent discharge. The relationship between the charges and fields inside a cloud and the probability of lightning initiation is not fully understood. It may be possible to monitor the charging processes in the storm cells (or storms) using these types of observations along with modeling.
[48] The concept of lightning flash suppression may also have applications in the area of lightning protection. For instance, in the presence of certain weather conditions, lightning may be triggered by firing a rocket unfurling a spool of wire toward a thundercloud [Rakov and Uman, 2007, ch.7]. Although it is known that triggered lightning is more successful when the flashing rate of the storm is relatively low, it is not currently known the degree to which triggered lightning may suppress natural activity (and thus protect against it), and such experiments may require a large number of repetitions. But natural lightning provides a highly repetitive laboratory for such a test.

[49] Acknowledgments. This work is supported by the Defense Advanced Research Project Agency under grant HR0011-10-1-0058P00001 to Stanford University. NLDN data are provided by Vaisala, Inc.

\section{References}

Berger, K., R. Anderson, and H. Kroninger (1975), Parameters of lightning flashes, Electra, 80, 223-237.

Chalmers, J. A., (1967), Atmospheric electricity, Pergamon.

Cummer, S. A., and U. S. Inan (1997), Measurement of charge transfer in sprite-producing lightning using ELF radio atmospherics, Geophys. Res. Lett., 24(14), 1731-1734.

Cummins, K. L., and M. J. Murphy (2009), An overview of lightning locating systems: History, techniques, and data uses, with an in-depth look at the U.S. NLDN, IEEE Trans. Electromag. Compatibility, 51(3), 499-518.

Cummins, K. L., E. P. Krider, and M. D. Malone (1998), The U.S. national ligthning detection network and applications of cloud-to-ground lightning data by electric power utilities, IEEE Trans. on Electromag. Compat., 40, 465-480.

Cummins, K. L., J. A. Cramer, W. A. Brooks, and E. P. Krider (2005), On the effect of land:sea and other earth surface discontinuities on lls-inferred lightning parameters, in VIII International Symposium on Lightning Protection.

Dennis, A. S. (1970), The flashing behavior of thunderstorms, J. Atmos. Sci., 27, 171-172.

Finke, U. (1998), Space-time correlations of lightning distributions, Monthly Weather Review, 127, 1850-1861.

Gopalakrishnan, V., S. D. Pawar, P. Murugavel, and K. P. Johare (2011), Electrical characteristics of thunderstorms in the Eastern part of India, J. Atmos. and Solar-Terres. Phys., 73, 1876-1882.

Hagenguth, J. H. (1951), The lightning discharge, Compendium of Meteorology, pp. 136-143.

Illingworth, A. J., and T. W. Wormell (1971), Electric field recovery after lightning, Nature Phys. Sc., 229, 213-214.

Jacobson, E. A., and E. P. Krider (1976), Electrostatic field changes produced by Florida lightning, J. Atmos. Sci., 33, 113-117.

Krehbiel, P. R., M. Brook, and R. A. McCrory (1979), An analysis of the charge structure of lightning discharges to the ground, J. Geophys. Res., $84,2432-2456$.

Kuettner, J. (1950), The electrical and meteorological conditions inside thunderclouds, Journal of Meteorology, 7, 322-332.

Lyons, W. A. (1996), Sprite observations above the U.S. high plains in relation to their parent thunderstorm systems, J. Geophys. Res., 101(D23), $29,641-29,652$.

Lyons, W. A., M. Uliasz, and T. E. Nelson (1998), Large Peak Current Cloud-to-Ground Lightning Flashes during the Summer Months in the Contiguous United States, Monthly Weather Review, 126, 2217, doi:10.1175/1520-0493(1998)126<2217:LPCCTG > 2.0.CO;2.

MacGorman, D. R., and W. D. Rust (1998), The Electrical Nature of Storms, Oxford University Press.

Mazur, V. (1982), Associated lightning discharges, Geophys. Res. Lett., 9(11), 1227-1230.

Mazur, V., P. R. Krehbiel, and X. M. Shao (1995), Correlated high-speed video and radio interferometric observations of a cloud-to-ground lightning flash, J. Geophys. Res., 100, 25,731-25,753.

Nag, A., et al. (2011), Evaluation of U.S. National Lightning Detection Network performance characteristics using rocket-triggered lightning data acquired in 2004-2009, J. Geophys. Res., 116(D02123), doi:10.1029/ 2010JD014,929.

Orville, R. E., G. R. Huffines, W. R. Burrows, R. L. Holle, and K. L. Cummins (2001), North American Lightning Detection Network (NALDN) First Results: 1998-2000, AGU Fall Meeting Abstracts, p. A59. 


\section{ZOGHZOGHY ET AL.: LIGHTNING FLASH SUPPRESSION}

Orville, R. E., G. R. Huffines, W. R. Burrows, and K. L. Cummins (2011), The North American lightning detection network (NALDN) - Analysis of flash data: 2001-09, Monthly Weather Review, 139, 1305-1322.

Peckham, D. W., M. A. Uman, and C. E. Wilcox (1984), Lightning phenomenology in the tampa bay area, J. Geophys. Res., 89(D7), 11,789-11,805.

Rakov, V. A., and M. A. Uman (2007), Lightning: Physics and Effects, Cambridge University Press.

Rakov, V. A., R. Thottappillil, and M. A. Uman (1992), Electric field pulses in $\mathrm{K}$ and $\mathrm{M}$ changes of lightning ground flashes, J. Geophys. Res., 97, 9935-9950.

Rhodes, C., and P. R. Krehbiel (1989), Interferometric observations of a single stroke cloud-to-ground flash, Geophys. Res. Lett., 16, 1169-1172.

Said, R. K., U. S. Inan, and K. L. Cummins (2010), Long-range lightning geo-location using a VLF radio atmospheric waveform bank, J. Geophys. Res., 115, D23108, doi:10.1029/2010JD013863.

Said, R. K., M. B. Cohen, and U. S. Inan (2013), Peak currents and incidence of land and oceanic lightning: Global observations by GLD360, manuscript in review with, J. Geophys. Res., doi:10.1029/2012JD019490.

Saraiva, A. C. V., M. M. F. Saba, J. O. Pinto, K. L. Cummins, E. P. Krider, and R. L. Holle (2010), On the variability of lightning characteristics over thunderstorm lifecycles, 21st International Lightning Detection Conference.

Stall, C. A., K. L. Cummins, E. P. Krider, and J. A. Cramer (2009), Detecting multiple ground contacts in cloud-to-ground lightning flashes, J. Atmos. Oceanic Tech., 26, 2392-2402.
Thottappillil, R., V. A. Rakov, and M. A. Uman (1990), K and M changes in close lightning ground flashes in Florida, J. Geophys. Res., 95, 18,631-18,640.

Vonnegut, B., O. H. Vaughan, M. Brook, and P. Krehbiel (1985), Mesoscale observations of lightning from space shuttle, Bull. Am. Met. Soc., 66(1), 20-29.

Williams, E., and Y. Yair (2006), The microphysical and electrical properties of sprite-producing thunderstorms, in Sprites Elves and Intense Lightning Discharges.

Williams, E. R. (1985), Large-scale charge separation in thunderclouds, J. Geophys. Res., 90, 6013-6025.

Wilson, C. T. R. (1916), On some determinations of the sign and magnitude of electric discharges in lightning flashes, Proc. Roy. Soc., 92, $555-574$.

Winn, W. P., and L. G. Byerley (1975), Electric field growth in thunderclouds, Q. J. R. Met. Soc., 101, 979-994.

Wormell, T. W. (1939), The effect of thunderstorms and lightning discharges on the Earth's electric field, Phil. Trans. Roy. Soc., 328, 249-303.

Yair, Y., R. Aviv, G. Ravid, R. Yaniv, B. Ziv, and C. Price (2006), Evidence for syncrhronicity of lightning activity in networks of spatially remote thunderstorms, J. Atmos. Sol.-Terr. Phys., 68, 1401-1415.

Yair, Y., R. Aviv, and G. Ravid (2009), Clustering and synchronization of lightning flashes in adjacent thunderstorm cells from lightning location networks data, J. Geophys. Res., 114(D09210), doi:10.1029/2008JD010,739. 